\title{
Arcillas Wealdenses en Cantabria (N. de España): Su Aprovechamiento Cerámico
}

\author{
L.A. DÍAZ RODRÍGUEZ y R. TORRECILLAS \\ Instituto Nacional del Carbón (CSIC). Dpto. de Química de Materiales \\ C/ Francisco Pintado Fe, 26, 33011 - Oviedo. España
}

\begin{abstract}
Se investiga la caracterización cerámica de los materiales arcillosos existentes en Cantabria (N. de España) pertenecientes a la facies Weald. Esta facies está englobada, dentro del Periodo Cretácico Inferior, en el denominado Grupo de Pas, que consta de dos formaciones: Formación Bárcena Mayor y Formación Vega de Pas (abarcando en Edad desde el Valanginiense Superior hasta el Barremiense). Las muestras tomadas (52 en total) dentro de estas dos secuencias deposicionales fueron sometidas a su caracterización granulométrica, mineralógica y química, para posteriormente, en las zonas favorables, realizar los oportunos ensayos tecnológicos. Los resultados obtenidos confirman su excelente viabilidad para la fabricación de productos cerámicos de la construcción en los sectores de ladrillos caravista y tejas y, sobre todo, como pavimentos y revestimientos de pasta roja.
\end{abstract}

Palabras clave: arcillas, facies Weald, Cantabria, arcillas comunes, caolines sedimentarios, arcillas plásticas, análisis tecnológicos, productos de gres, cerámica roja.

\section{Wealden clays from Cantabria (N.Spain): Their ceramic use}

Several clay sediments from the Weald facies (Barcena Mayor Formation, Vega de Pas Formation, including Upper Valanginian to Barremian) belonging to the Pas Group (Lower Cretaceous Period) from Cantabria (N. Spain) have been studied. Fifty two samples were extracted from several outcroppings and granulometric, mineralogic and chemical analyses were carried out. Samples from the clay-rich zones were technologically characterized and the results indicate that they could be used as red stoneware ceramic products (facing bricks, roof, and floor tiles).

Keywords: clays, Weald facies, Cantabria (N. Spain), common clays, sedimentary kaolins, fire clay, technological analysis, red stoneware products.

\section{INTRODUCCIÓN}

La Comunidad Autónoma de Cantabria es una región favorecida geológicamente por su elevado contenido en materiales arcillosos, sobre todo aquellos que contienen arcillas comunes, con formaciones geológicas muy potentes, $\mathrm{y}$, en menor proporción, del tipo "caolines secundarios" (1) (2). Las arcillas wealdenses constituyen una de las mayores acumulaciones arcillosas que se extienden por toda la Comunidad, siendo la materia prima fundamental de la que se abastecen, en la actualidad, las principales tejeras que se mantienen en funcionamiento.

El "Wealdense" ha sido estudiado a escala de la Península Ibérica por Aguayo y Martín Vivaldi (3) y Caballero y Martín Vivaldi (4), donde se pueden encontrar datos mineralógicos e hipótesis genéticas acerca de la Cuenca Wealdense de Cantabria. La geología de esta cuenca ha tenido diversas interpretaciones geológicas y un flujo continuo de nuevos datos desde que González Linares, en 1876, demostró la existencia de materiales wealdenses en la Península Ibérica y concretamente en Cantabria. Así, entre otros estudios estratigráficos, y a partir de la década de los años 70, se puede citar los de Ramírez del Pozo y Aguilar (5), Brenner (6), Salomón (7) y Pujalte (8).

En la actualidad, el Wealdense (o Complejo PurbeckWeald) de Cantabria se considera que está constituido por dos secuencias deposicionales mayores, a las que se les asigna el rango de Grupo: Grupo Cabuérniga y Grupo Pas (9). Las facies Weald objeto del estudio en este trabajo se encuentran dentro del Grupo Pas, que consta de dos formaciones, del periodo Cretácico Inferior, cuya edad abarca desde el Valanginiense Superior hasta el Barremiense, que son la Formación Bárcena Mayor y la Formación Vega de Pas.

Desde el punto de vista cerámico, se pueden citar diversos trabajos más generales (2) (10) (11)(12)(13) sobre las arcillas wealdenses de Cantabria y su comportamiento tecnológico. Aunque esta región tiene potentes afloramientos wealdenses, el número de explotaciones activas existentes se reduce en la actualidad a tres: una en la Navas de Turujal y otra en Virgen de la Peña, ambas en los alrededores de Cabezón de la Sal y pertenecientes al mismo propietario, Cerámica de Cabezón S. A., y la última Tejerías La Covadonga S.A., que explota una cantera en los alrededores de Parbayón.

En este trabajo, se analizan los principales indicios y depósitos de arcillas con facies Weald en Cantabria, efectuándose estudios granulométricos, mineralógicos, químicos y tecnológicos, que indican el aprovechamiento más favorable de este tipo de materiales arcillosos. 


\section{MARCO GEOGRÁFICO Y GEOLÓGICO}

La Comunidad Autónoma de Cantabria ha sido tradicionalmente dividida en dos grandes áreas naturales: la Marina y la Montaña. La Marina es la zona situada a lo largo de la franja costera, con un relieve y clima moderados y una alta densidad de población. La Montaña, que da nombre a los habitantes de estas tierras, abarca prácticamente los $2 / 3$ de la Región y posee un relieve abrupto, con un clima más riguroso y una densidad de población más escasa.

Estas dos grandes áreas pueden ser subdivididas, a grandes rasgos, en tres conjuntos o franjas que abarcan diez comarcas con una entidad propia bastante bien definida (14), encontrándose los principales indicios y depósitos estudiados en la denominada Franja Litoral Costera, que es una zona de unos $20 \mathrm{~km}$ de anchura y limitada al Norte por el mar Cantábrico y al Sur por la falla del Escudo de Cabuérniga y tanto al Este como al Oeste por Asturias y Vizcaya respectivamente (Fig.1A y C). Geológicamente, Cantabria pertenece, dentro del Dominio Peri-Asturiano, a la parte más occidental de la denominada Región Vasco-Cantábrica (15) (Fig.1A).

La facies Weald objeto de este estudio se encuentra dentro del Grupo Pas, que consta de dos formaciones: La Formación Bárcena Mayor y la Formación Vega de Pas (Fig.1B).

La Formación Bárcena Mayor (Valangeniense SuperiorHauteriviense Inferior) está constituida mayoritariamente por areniscas blanquecino-amarillentas, con intercalaciones lentejonares de lutitas grises y rojas, siendo la única unidad litoestratigráfica dentro del Wealdense de Cantabria con indicios de caolín en sus estratos (arenas caoliníferas) (2).

La Formación Vega de Pas (Hauteriviense SuperiorBarremiense) se sitúa sobre la Formación anterior y consta de dos miembros: el inferior o "Capas de Vivíparus" y el superior o “Capas Rojas". El primero está constituido por lutitas negras, limolitas grises verdosas y areniscas calcáreas lumaquélicas (gasterópodos y lamelibranquios). El miembro "Capas Rojas" consta fundamentalmente de limolitas arcillosas rojas masivas, con algún tono verdoso, y, en menor proporción, areniscas cremas y grisáceas. En la Figura 1C, se puede contemplar la distribución de las explotaciones y los indicios estudiados.

\section{METODOLOGÍA EXPERIMENTAL}

Las muestras obtenidas a lo largo de los recorridos de campo (52 en total, de las que 10 pertenecen a la Formación Bárcena Mayor y el resto a la Formación Vega de Pas) fueron sometidas, en general, a dos tipos de análisis. En una primera fase, todas las muestras fueron sometidas a los correspondientes análisis granulométricos, mineralógicos y químicos. Con posterioridad, la adecuada potencia de la unidad litoestratigráfica muestreada junto con los análisis favorables nos proporcionaron los datos necesarios para poder elegir las muestras a ensayar tecnológicamente.

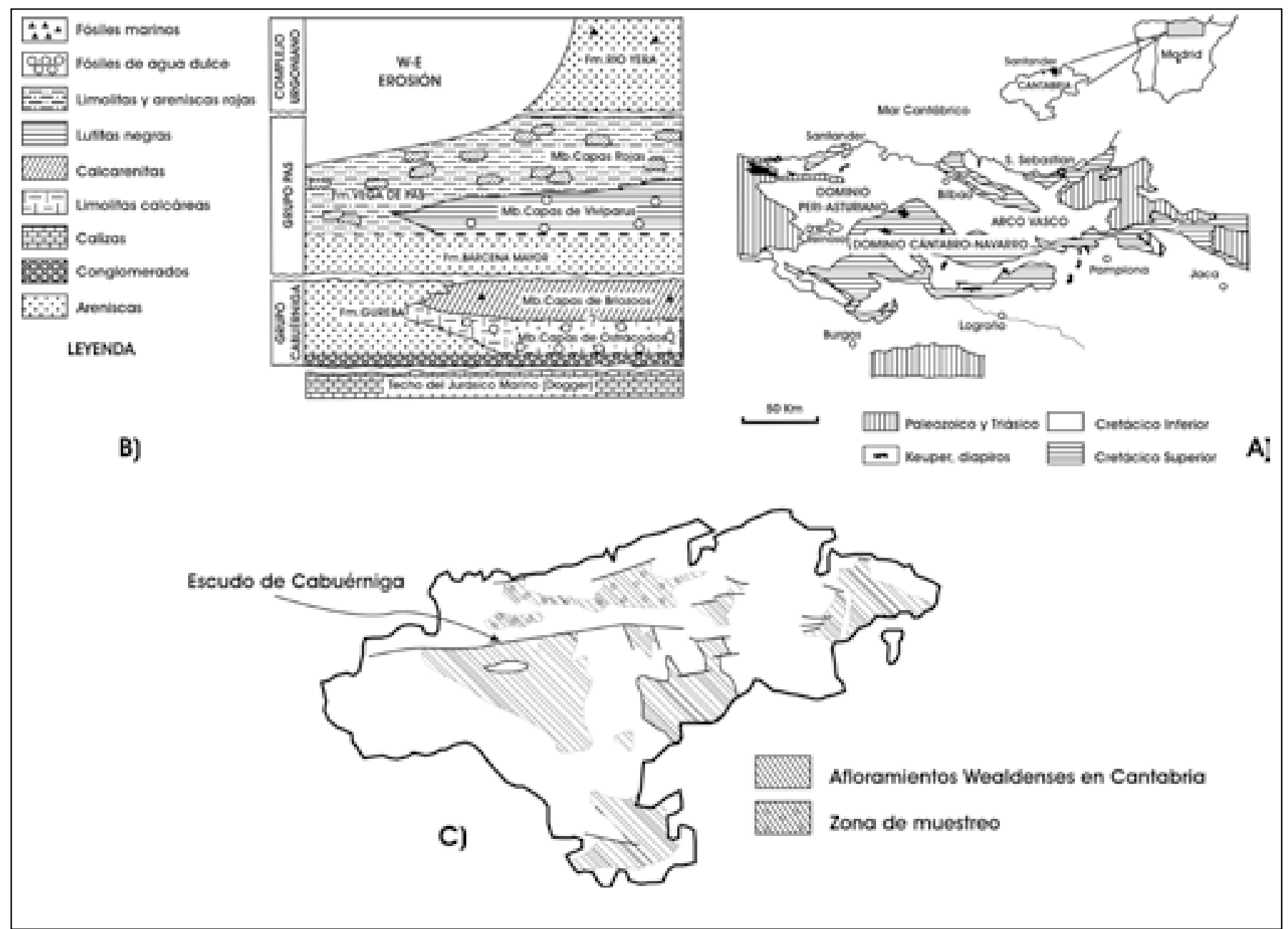

Fig. 1- A) Marco geológico y geográfico de la cuenca Vasco-Cantábrica. B) Corte E-W en Cantabria de la columna litoestratigráfica que abarca desde el Jurásico Superior hasta el Cretácico Inferior (8). C) Distribución geográfica de los afloramientos wealdenses en Cantabria. 
Los análisis granulométricos se realizaron mediante tamizado en húmedo desde $4 \mathrm{~mm}$ hasta la abertura de malla de 80 micras y, para tamaños inferiores, se empleó un equipo granulométrico de medición por láser (Coulter, LS 130). Los intervalos considerados como fracción arena, limo y arcilla se basan en los trabajos de Wentworth (16):

$$
\begin{aligned}
& \text { arena }>62 \mu \mathrm{m} \\
& 4 \mu \mathrm{m}<\operatorname{limo}<62 \mu \mathrm{m} \\
& \text { arcilla }<4 \mu \mathrm{m}
\end{aligned}
$$

El estudio mineralógico se llevó a cabo por difracción de rayos X, empleando un equipo Siemens modelo D5000 con las siguientes condiciones de trabajo: anticátodo de cobre refrigerado por agua trabajando a $30 \mathrm{~mA}$ y $40 \mathrm{kV}$. El barrido analítico del "Todo-Uno" se realizó entre $2-65^{\circ}(2 \theta)$, mientras que para los agregados orientados, entre 2 y $40^{\circ}(2 \theta)$. El tamaño de paso del barrido fue de $0,02^{\circ}$ y el tiempo de paso de $1 \mathrm{~s}$. De las muestras tomadas, se obtuvieron cuatro difractogramas: 1) Todo-Uno, 2) agregado orientado, 3) agregado orientado y tratado térmicamente a $550^{\circ} \mathrm{C} / 2 \mathrm{~h}$ y 4 ) saturado en etilenglicol durante $24 \mathrm{~h}$ (17), con el objeto de caracterizar perfectamente tanto los minerales arcillosos como los no arcillosos.

Las muestras cuarteadas fueron analizadas químicamente por espectrometría de fluorescencia de rayos $\mathrm{X}$ en un equipo Siemens SR3000. Se analizaron en forma de perlas, utilizando una perladora Philips y empleando $\mathrm{Li}_{2} \mathrm{~B}_{4} \mathrm{O}_{7}$ como fundente y $\mathrm{LiBr}$ como despegante. Los elementos analizados por esta técnica fueron $\mathrm{Si}, \mathrm{Al}, \mathrm{Fe}, \mathrm{Ca}$, Ti, Mn y K. Otros elementos, como el $\mathrm{Mg}$ y $\mathrm{Na}$, por espectrofotometría de absorción atómica, y el contenido en sulfatos, por métodos gravimétricos.

La caracterización físico-tecnológica de las arcillas de facies Weald de Cantabria se llevó a cabo tanto en las materias primas en sí como en los productos cocidos obtenidos con las mismas. Se estudiaron las propiedades tanto en crudo como en cocido de las muestras seleccionadas (9 en total):

1) Propiedades en crudo:

a) Plasticidad (límites de Atterberg), según las normas UNE 7-377 y 7-378. El límite líquido se determinó utilizando la "cuchara de Casagrande" y el plástico calculando el contenido de agua de cilindros de arcilla amasada de unos $3 \mathrm{~mm}$ de diámetro, en el momento en que éstos comienzan a agrietarse por la pérdida de agua sobre un molde de escayola.

b) Resistencia a la flexión de probetas en verde. El equipo de análisis es una máquina universal de ensayos Instron modelo 8562. Se ensayaron tres probetas y se obtuvo el valor promedio.

c) Agua de amasado y contracción de secado.

2) Propiedades en cocido:

a) Diagramas de gresificación (contracción lineal y absorción de agua). Las probetas fueron prensadas isostáticamente (200 $\mathrm{MPa}$ ) y se sometieron a un ciclo de calentamiento de tipo industrial (Tabla I). El tiempo de estancia a la temperatura final fue de una hora.

TABLA I. CICLO INDUSTRIAL DE CALENTAMIENTO
\begin{tabular}{|c|c|c|}
\hline $\begin{array}{c}\text { RAMPAS } \\
\left({ }^{\circ} \mathrm{C} / \mathrm{min}\right)\end{array}$ & $\begin{array}{c}\text { INTERVALO DE } \\
\text { TEMPERATURAS }\left({ }^{\circ} \mathrm{C}\right)\end{array}$ & $\begin{array}{c}\text { TIEMPO EMPLEADO } \\
(\mathrm{h})\end{array}$ \\
\hline 1 & $25-250$ & 4,17 \\
\hline 3 & $250-550$ & 1,67 \\
\hline 2 & $550-950$ & 3,33 \\
\hline 3,5 & $950-\mathrm{T}$. final & - \\
\hline 3 & T.final-750 & - \\
\hline 1 & $750-550$ & 3,33 \\
\hline Caida libre & $550-25$ & - \\
\hline
\end{tabular}

b) Resistencia a la flexión de probetas después de un tratamiento térmico. Se ensayaron probetas cilíndricas extrusionadas de $1,5 \mathrm{~cm}$ de diámetro y cocidas a dos temperaturas: 1050 y $1100^{\circ} \mathrm{C}$. También se obtuvo un valor promedio de tres probetas ensayadas.

c) Coeficiente de dilatación. Este valor se analizó mediante un dilatómetro de la casa Netzch modelo 402 con un portamuestras de alúmina y en atmósfera oxidante. Se determinó la dilatación que experimenta la pieza cocida a distintas temperaturas y se calcularon los distintos coeficientes de dilatación como pendiente entre dos puntos de temperatura determinados $\left(300-500^{\circ} \mathrm{C}\right)$.

d) Ensayo de corazón negro. Las probetas fueron prensadas isostáticamente a $200 \mathrm{MPa}$ y posteriormente fueron sometidas a ciclos de calentamiento muy rápidos $\left(30^{\circ} \mathrm{C} / \mathrm{min}\right.$ hasta $\left.900^{\circ} \mathrm{C}\right)$ para observar en su fractura o corte transversal la mancha central que podía producirse.

Además, se llevó a cabo un estudio del comportamiento térmico del material arcilloso de la Formación Vega de Pas mediante un microscopio de calefacción de la casa Leica $\left(1750^{\circ} \mathrm{C}\right)$ con un equipamiento Novocontrol Mitronic II. Para ello, la muestra objeto de análisis se molturó y tamizó por debajo de 63 micras humectándose a continuación con agua destilada al $5 \%$ para obtener un cilindro de $3 \mathrm{~mm}$ de diámetro y $3 \mathrm{~mm}$ de altura.

\section{RESULTADOS Y DISCUSIÓN}

En la Figura 2, se puede contemplar la representación gráfica en el diagrama de Shepard(18) los resultados de las granulometrías obtenidas de las muestras de las formaciones Bárcena Mayor y Vega de Pas. En líneas generales, todas las muestras analizadas son en su mayoría de naturaleza limolítica y limolítica arcillosa. En algunas muestras, fundamentalmente en niveles areniscosos, el tamaño de partícula predominante es de tipo arena.

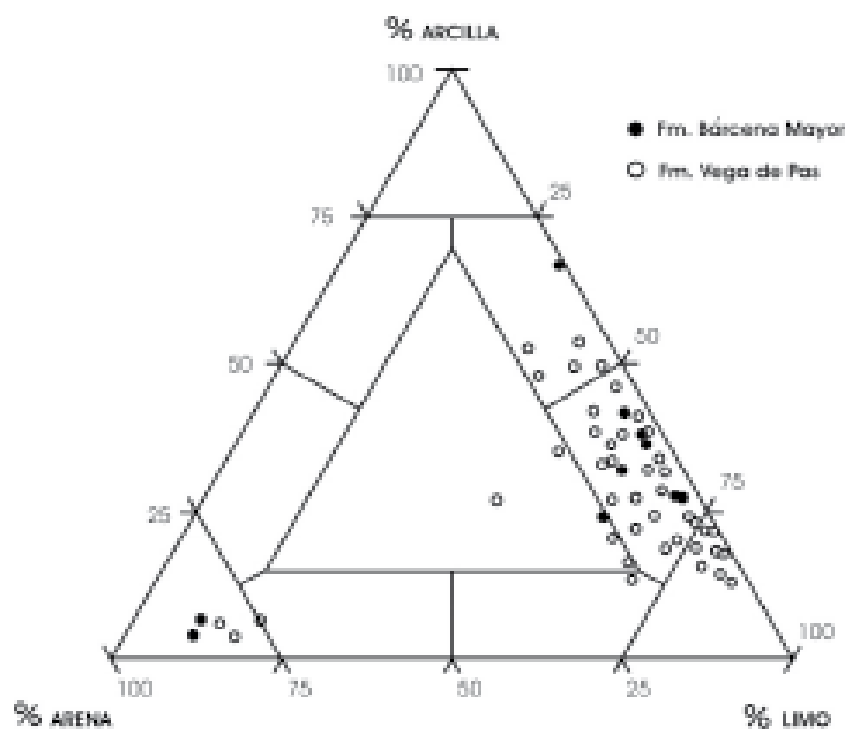

Fig. 2- Distribución granulométrica de las muestras analizadas, según el diagrama de Shepard (18).

Los resultados de los estudios mineralógicos efectuados se pueden contemplar en la Tabla II. En general, el cuarzo es el mineral mayoritario dentro de los minerales no arcillosos 
TABLA II. RESULTADOS DE LA COMPOSICIÓN MINERALÓGICA DE LAS MUESTRAS TOMADAS OBTENIDOS POR DIFRACCIÓN DE RAYOS X. SE EXPONEN LOS MINERALES PRINCIPALES PROMEDIO DEL TODO-UNO Y TAMBIÉN LOS ACCESORIOS Y TRAZAS. TAMBIÉN SE EXPONEN LOS MINERALES PRINCIPALES DE LA FRACCIÓN ARCILLOSA

\begin{tabular}{|c|c|c|c|c|}
\hline & Min. Principal & Accesorios y Tranos & Min. Principal & Accesorios y Tranas \\
\hline $\begin{array}{l}\text { Formación } \\
\text { Bárcena Mayor (10) }\end{array}$ & Cuarzo & Feldespatos y Plagioclasas & Caolinita, Mica & Mica \\
\hline $\begin{array}{l}\text { Formación } \\
\text { Wega de Pas (42] }\end{array}$ & Cuarzo & Feidespatos, Calcita & Mica, Caolinita & Coolinila, Vermiculita, Esmectita, Clonite \\
\hline
\end{tabular}

$[5]=$ numero de muestros analizadas

TABLA III. RESULTADOS DE LOS ANÁLISIS QUÍMICOS EFECTUADOS SOBRE EL CONJUNTO DE MUESTRAS TOMADAS. VALORES PROMEDIADOS.

\begin{tabular}{|c|c|c|c|c|}
\hline & Min. Principal & Accesorios y Trasos & Min. Mincipal & Accesorios y Trazos \\
\hline $\begin{array}{l}\text { Formación } \\
\text { Bárcena Mayor (10) }\end{array}$ & Cuarzo & Feldespatos y Plagioclasas & Caolinita, Mica & Mica \\
\hline $\begin{array}{l}\text { Formación } \\
\text { Wega de Pas (42) }\end{array}$ & Cuarno & Feldespatos, Calcita & Mica, Caolinita & Coolinitha, Vermiculita, Esmoctita, Clonitc \\
\hline
\end{tabular}

[5]- numero de mueltros analizadas

TABLA IV. RESULTADOS DE LOS ANÁLISIS TECNOLÓGICOS EFECTUADOS SOBRE LAS NUEVE MUESTRAS SELECCIONADAS (VALORES PROMEDIO).

\begin{tabular}{|c|c|c|c|c|c|c|c|c|c|c|c|c|c|c|c|}
\hline & \multicolumn{4}{|c|}{ Plasticidod [\$S] } & \multicolumn{3}{|c|}{ Besitsencia $[\mathrm{kg}(\mathrm{cm})$} & \multirow{2}{*}{$\begin{array}{l}\text { Agua C } \\
\text { de } \\
\text { amosodo }\end{array}$} & \multirow{2}{*}{$\begin{array}{l}\text { Controcción } \\
\text { de }\end{array}$} & \multicolumn{3}{|c|}{ Controceión Ineal $(\$)$} & \multicolumn{3}{|c|}{ Absorcibn en ogua (\$) } \\
\hline & & L. Liquido & L. Plastico & 18 & 5000 & $1075^{\circ} \mathrm{C}$ & $1125^{\circ} \mathrm{C}$ & & & $1075^{\circ} \mathrm{C}$ & $1125^{\circ} \mathrm{C}$ & $1175^{\circ} \mathrm{C}$ & $1075 n c$ & $1125^{\circ} \mathrm{C}$ & $1175^{\circ} \mathrm{C}$ \\
\hline formacion & $\bar{x}=$ & 30,00 & 21.40 & 9.20 & 46 & 381.50 & 482 & 19 & 4.70 & 3.20 & 5.70 & 5.95 & 7.10 & 1.90 & 0.90 \\
\hline Barcena Mayoe (2) & $a=$ & 5,52 & 1,84 & 3.68 & s.s6 & 41,72 & 28.87 & 2.26 & 0.42 & 0,12 & 0,02 & 0.03 & 0,10 & 0,03 & 0.01 \\
\hline formacion & $\bar{x}=$ & 28.90 & 213 & 7.57 & 34,12 & 341.71 & 447.71 & 18.91 & 5.01 & 2.99 & 5.76 & 7.01 & 7.99 & 3.46 & 0.73 \\
\hline Vega de Ras ( 7 ) & $a=$ & 4,34 & 2.45 & 1,63 & 13.9 & $\$ 2,62$ & 79.3 & 2.12 & 0.09 & 1.34 & 0.92 & 0.59 & 1.99 & 2.19 & 0.22 \\
\hline
\end{tabular}

(5) = numeno de mueition fomodas

$x=$ volor medio

existiendo también como accesorios feldespatos (K-Na-Ca), $\mathrm{y}$, en algunas muestras, hematites y calcita. En cuanto a los minerales arcillosos, predominan la asociación caolinita-mica (tipo illita) o viceversa. El contenido en minerales del grupo del caolín es más abundante en los estratos de la Formación Bárcena Mayor y su proporción va disminuyendo al ascender de muro a techo en la escala geológica (Formación Vega de Pas), como así señala Díaz Rodríguez (12). Como minerales arcillosos accesorios aparecen los de los grupos de la clorita, la esmectita y la vermiculita. Los minerales arcillosos cloríticos van asociados al color verde de los estratos presentes en la Formación Vega de Pas.

Los análisis químicos efectuados pueden contemplarse en la Tabla III, estando totalmente relacionados con los análisis mineralógicos. El contenido en $\mathrm{Al}_{2} \mathrm{O}_{3}$ es moderado con valores máximos que van desde el $30 \%$ en peso, para algunos estratos arcillosos de la Formación Bárcena Mayor, hasta valores en torno al $24 \%$ en peso (21,45\% de promedio) de la Formación Vega de Pas. Los óxidos de hierro se presentan en una proporción significativa (con valores entre el 5 y $8 \%$ en peso), siendo los principales responsables de la coloración roja de los productos cocidos junto con la atmósfera oxidante de cocción. El resto de óxidos aparece de forma regular y en unos porcentajes proporcionados, en general, por debajo del $1 \%$ en peso, con excepción del contenido en óxido de potasio (valores promedio en torno al 5\%) proveniente de la abundancia de minerales arcillosos del grupo de las micas.

Los resultados de los análisis físico-tecnológicos llevados a cabo sobre las muestras escogidas se pueden contemplar en la Tabla IV.

Los valores de plasticidad (Tabla IV) son, en general, bastantes bajos, estando el índice de plasticidad por debajo del valor 10. Las muestras procedentes de la Formación Bárcena Mayor son algo más plásticas que las de las Formación Vega de Pas, que presenta una granulometría predominante de tamaño limolítica-arcillosa.

La resistencia mecánica de las probetas en crudo es también bastante baja y está relacionada directamente con las bajas plasticidades obtenidas. Los valores encontrados se hallan por debajo del los $50 \mathrm{~kg} / \mathrm{cm}^{2}$. Los valores más altos los presentan las muestras pertenecientes a la Formación Bárcena Mayor (Tabla III), que suelen poseer una buena compactación en crudo. En cuanto a la resistencia mecánica en cocido, los valores obtenidos (Tabla III) son muy altos para ambas formaciones, superando los $300 \mathrm{~kg} / \mathrm{cm}^{2}$ para la temperatura de $1075^{\circ} \mathrm{C}$. 

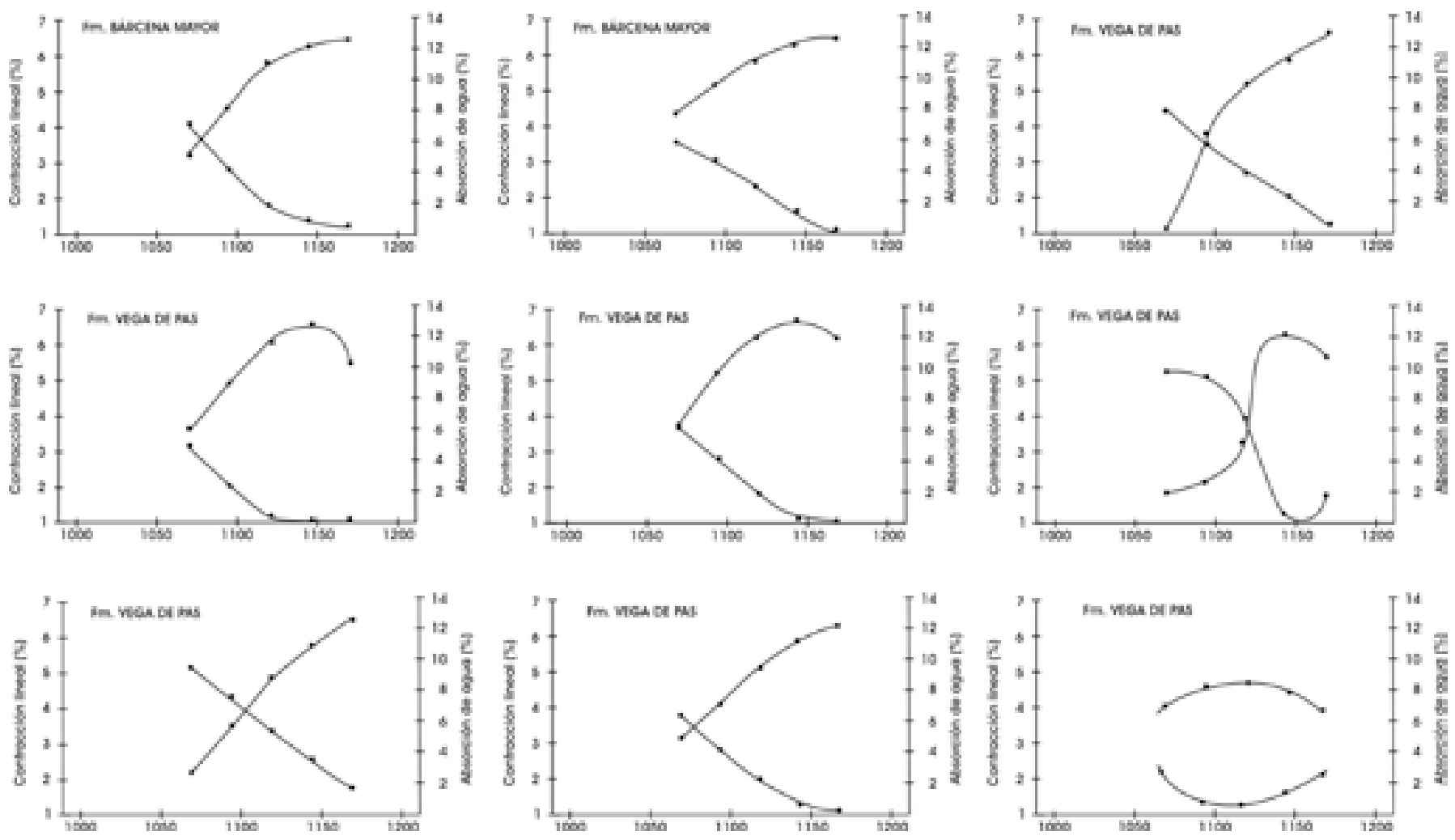

Fig. 3- Diagramas de gresificación de las nueve muestras seleccionadas. En el eje $\mathrm{X}$ se representa la temperatura en ${ }^{\circ} \mathrm{C}$.

En la Figura 3, se puede apreciar los diagramas de gresificación de las muestras seleccionadas (9 en total). Los diagramas obtenidos confirman su carácter gresificable, con unos rangos de cocción muy estrechos, donde, pasado un determinado valor, las piezas hinchan por la formación de gases ocluidos en el interior de las mismas, por la descomposición de la hematites $\left(\mathrm{Fe}_{2} \mathrm{O}_{3}\right)$ para formar magnetita $\left(\mathrm{Fe}_{3} \mathrm{O}_{4}\right)$ con pérdida de oxígeno (19). En líneas generales, las muestras de ambas formaciones poseen valores de absorción de agua en torno al $3 \%$ cuando se alcanzan temperaturas de $1100^{\circ} \mathrm{C}$. Las muestras de la Formación Bárcena Mayor (ver Tabla III) presentan unos valores de cocción algo más amplios

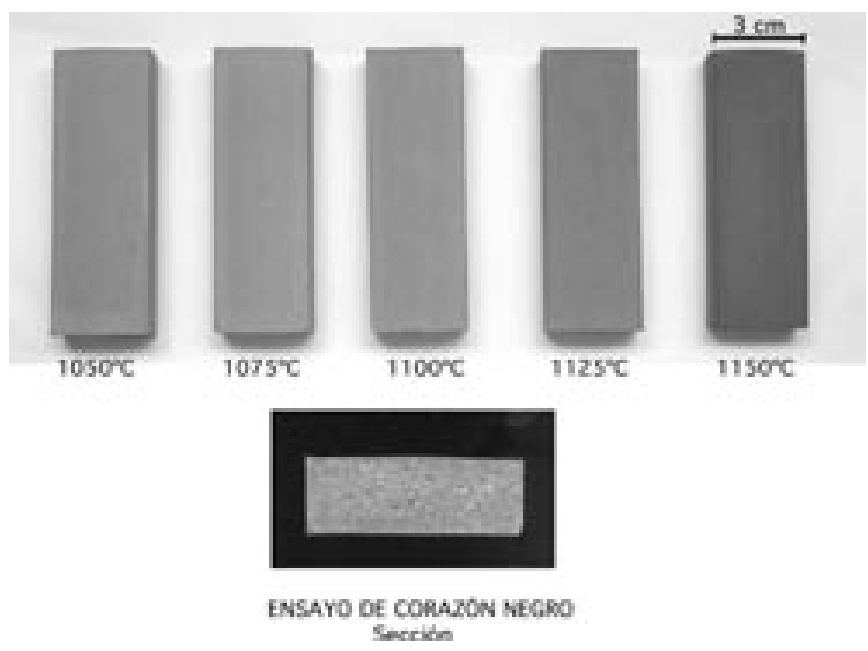

Fig. 4- Aspecto "de visu" que presentan las piezas cocidas a distintas temperaturas de una de las muestras perteneciente a la Formación Vega de Pas, junto con el ensayo de corazón negro

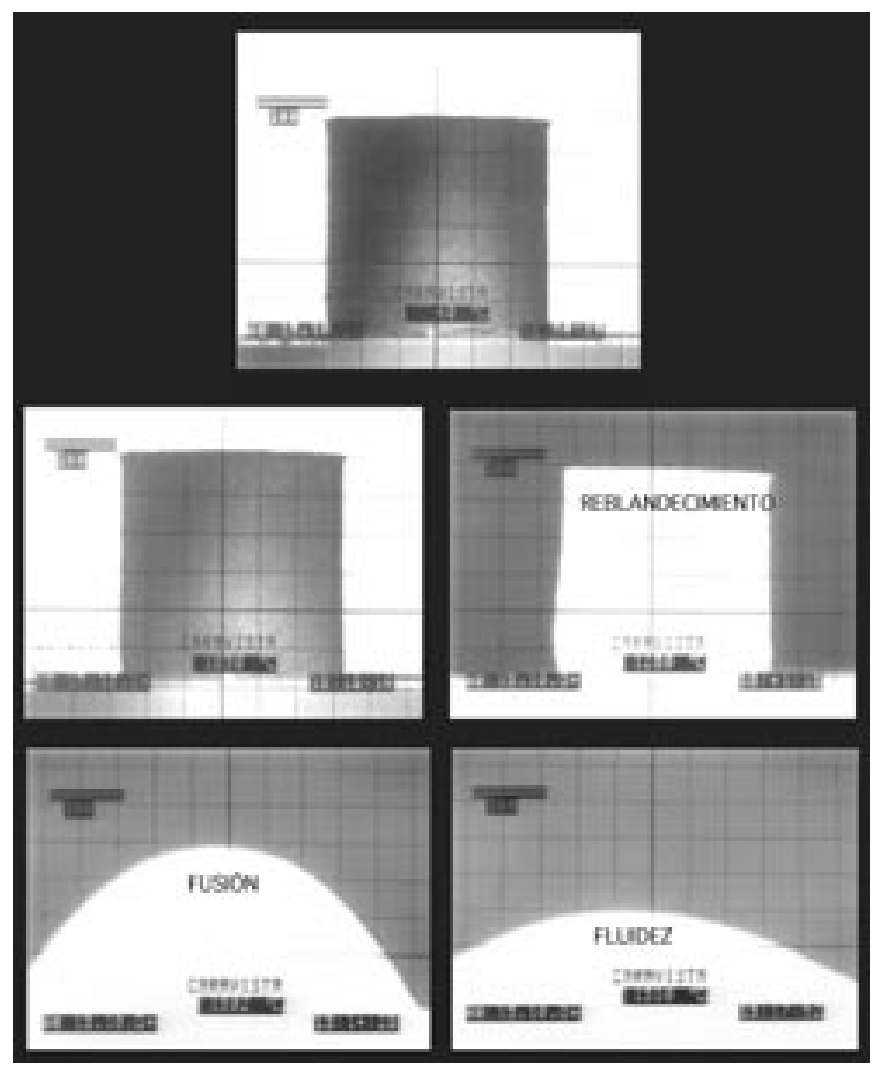

Fig. 5- Muestra de la Formación Vega de Pas bajo microscopía de calefacción. Se aprecian los tres puntos característicos a las siguientes temperaturas: $1280^{\circ} \mathrm{C}$ (reblandecimiento), $1502^{\circ} \mathrm{C}$ (fusión) y $1530^{\circ} \mathrm{C}$ (fluidez). 
debido a su composición mineralógica con mayor contenido de minerales del grupo del caolín y una temperatura de gresificación ligeramente superior a las muestras procedentes de la Formación Vega de Pas.

En la Figura 4, se puede contemplar el aspecto que presentan algunas de las probetas cocidas a distintas temperaturas (Formación Vega de Pas) y el resultado del ensayo de corazón negro efectuado donde no se aprecia ninguna mancha oscura en el interior de la pieza.

Mediante microscopía de calefacción, se efectuó un estudio de los puntos característicos de reblandecimiento, fusión y fluidez de un material arcilloso de la Formación Vega de Pas (Fig.5). Como se puede ver en dicha Figura 5, el punto de reblandecimiento comienza a $\operatorname{los} 1280^{\circ} \mathrm{C}$, el de fusión se alcanza a los $1502^{\circ} \mathrm{C}$ y el de fluidez, unos $28^{\circ} \mathrm{C}$ más arriba, a $1530^{\circ} \mathrm{C}$.

\section{CONCLUSIONES}

Las arcillas de la facies Weald de Cantabria desde el punto de vista cerámico pertenecen a los grupos de las arcillas comunes y de los caolines. La Formación Bárcena Mayor es la que alberga los caolines sedimentarios y, en concreto, arenas caoliníferas e interestratos arcillosos de tipo fire-clay (subtipo plastic clay) de colores grises abigarrados y de escasa potencia. La Formación Vega de Pas, sin embargo, posee arcillas comunes de color rojo con amplios afloramientos que la configuran como una Formación con un gran potencial para los sectores industriales de la cerámica roja. Los niveles arcillosos de la Formación Bárcena Mayor e incluso algunos de sus capas arenosas pueden ser utilizados en los sectores de loza y porcelana, aunque la potencia de dichos niveles es escasa, lo que dificulta su posible explotación. La composición mineralógica de los materiales arcillosos de esta Formación pertenece a la asociación caolinita-mica por este orden de abundancia. Son arcillas de cocción clara con alta proporción de cuarzo como mineral desgrasante y con posibilidad de ser empleadas en los productos de pasta blanca donde no se requiere un grado de blancura muy elevado, especialmente en la fabricación de pavimentos gresificados y revestimientos porosos por prensado.

Por el contrario, los materiales arcillosos rojos de la Formación Vega de Pas son muy adecuados para los sectores industriales de la cerámica roja: ladrillos, tejas, pavimentos y revestimientos; fundamentalmente para productos obtenidos por prensado dado el tipo de granulometría y la escasa plasticidad que poseen estos materiales. En este caso, la mineralogía de los materiales arcillosos de estas arcillas rojas es del tipo mica-caolinita, predominando las fracciones illíticas.

En general, los materiales arcillosos de la facies Weald de Cantabria poseen bajas plasticidades, siendo materiales muy recomendables para la fabricación por prensado de productos gresificables tanto para ladrillos caravista como pavimentos, dado que se alcanzan bajas absorciones de agua a temperaturas de cocción no muy altas $\left(1075-1100^{\circ} \mathrm{C}\right)$.

\section{BIBLIOGRAFÍA}

1. L.A. Díaz Rodríguez y R. Torrecillas. “Arcillas cerámicas: una revisión de sus distintos tipos, significados y aplicaciones". Bol. Soc. Esp. Cerám. V., 41, [5], 459-470 (2002)

2. L.A. Díaz Rodríguez, V. Calderón Almena y M. Peralta Vaquero. “Tipos de arcillas en Cantabria“. II Congreso Español de Geología, Granada, T. 2, 267-270 (1988).

3. F. López Aguayo y J.L. Martín Vivaldi. “Mineralogía de las arcillas de la facies Wealdense española. I. Antecedentes, métodos y materiales. II. Cuencas Norte, Levantina y Bética. III. Cuenca Asturiana, discusión y conclusiones". Estudios Geológicos, 29, 389-437 (1973).

4. M.A. Caballero y J.L. Martín Vivaldi. “Estudio mineralógico y genético de la fracción fina del Trías español“. Memoria del Instituto Geológico y Minero de España, T.87, 227 pp. (1975)

5. J. Ramírez del Pozo y M.J. Aguilar Tomás. “Consideraciones sedimentológicas y paleogeográficas de las facies Purbeck y Weald de la cubeta de SantanderBurgos“. Estudios Geológicos, 28, 173-192 (1973).

6. P. Brenner. "Ostracoden und Charophyten des Spanischen Wealden (Systematik, Okologie, Stratigraphie, Paläegeographie)“. Paleontographica Abt. A., 152, 113-201 (1976).

7. J. Salomón. “Les formations continentales du Jurassique superior-Crétacé inferieur (Espagne du Nord, Chaines Cantabrica et NW Iberica“. Memoires Géologiques de l’Université de Dijon, 6, 227 pp (1982).

8. V. Pujalte. "The "Wealden" basin of Santander". Abstracts from $6^{\text {th }}$ European Regional meeting of I.A.S., Excursion Guidebook (Lleida, Spain), Ed. By M.D. Mila \& J. Rosell, April, 351-371 (1985).

9. V. Pujalte. "El tránsito Jurásico-Cretácico, Berriasiense, Valanginiense, Hauteriviense y Barremiense“. En: El Cretácico en España. Capítulo Tercero. Ed. Univ. Complutense, Madrid, 51-63 (1982).

10. G. Gómez Moreno, L.A. Díaz Rodríguez, V. Calderón Almena y M. Peralta Vaquero. "Caracterización tecnológica de las arcillas de Cantabria (N. De España) y su aprovechamiento industrial“. VIII Congreso Internacional de Minería y Metalurgia, Oviedo, T. 6, 553-560 (1988).

11. L.A. Díaz Rodríguez. "Estudio general de los depósitos arcillosos en Cantabria (N. De España): Su uso potencial“. Boletín Geológico y Minero, V. 101-2, 253-269 (1990).

12. L.A. Díaz Rodríguez. "Weald facies from Cantabria (N. Spain): The exploitation of their ceramic resources". Fourth Euro-Ceramics, Rimini, Italy, edited by C. Palmonari, Faenza Editrice, Vol. 11, 27-33 (1995).

13. Instituto Geológico y Minero de España, Mapa Geológico - Minero de Cantabria (1:100.000). Servicio de Publicaciones IGME.133 pp. (1990)

14. A. Cendrero, J.R. Díaz de Terán, E. Flor, E. Francés, J.R. González Lastra, y J.M. Martínez Incera. “ Guía de la Naturaleza de Cantabria “. Ed. Librería Estudio, Santander, 256 pp. (1986).

15. P. Feuillee and P. Rat. "Structures et paléogéographies pyréneo cantabriques". In: Histoire Structurale du Golfo de Gascogne. Ed. By Technip (París), 1- 48 (1971).

16. C. K. Wentworth. "A scale of grade and clase terms for clastic sediments". J. Geol., 30, 377-392 (1922).

17. G. Brunton. "Vapor pressure glycolation of oriented clay minerals". Amer Mineral., 40, 124-126 (1955).

18. F.P. Shepard “ Nomenclature based on sand-silt-clay ratios “. J. Sed. Petrol., 24, 151-158 (1954).

19. A. Escardino Benlloch, J.L. Amoros Albaro, y J.E. Enrique Navarro. “ Estudio de pastas de gres para pavimentos“. Bol. Soc. Esp. Cerám. V., 20, [1], 17-24 (1981).

Recibido: 01.04 .05

Aceptado: 06.07.05 